\title{
Boron influence on bandgap and photoluminescence in BGaN grown on AIN
}

\author{
Cite as: J. Appl. Phys. 127, 165703 (2020); doi: 10.1063/1.5140413 \\ Submitted: 29 November 2019 . Accepted: 11 April 2020 . \\ Published Online: 29 April 2020
}

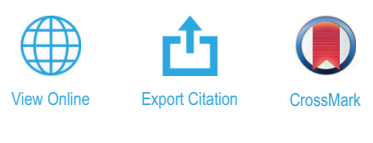

\author{
E. Zdanowicz, ${ }^{1,2, a)}$ (iD D. lida, ${ }^{3}$ (D) L. Pawlaczyk, ${ }^{4}$ (D) J. Serafinczuk, ${ }^{2,4}$ (iD R. Szukiewicz, ${ }^{2,5}$ (iD R. Kudrawiec, ${ }^{1,2}$ (iD \\ D. Hommel, 2,5 (D) and K. Ohkawa ${ }^{3}$ (D)
}

\begin{abstract}
AFFILIATIONS
${ }^{7}$ Department of Semiconductor Materials Engineering, Wrocław University of Science and Technology, Wyb. Wyspiańskiego 27, 50-370 Wrocław, Poland

${ }^{2}$ ŁUKASIEWICZ Research Network-PORT Polish Center for Technology Development, Stabłowicka 147, 54-066 Wrocław, Poland

${ }^{3}$ Computer, Electrical and Mathematical Sciences and Engineering Division, King Abdullah University of Science and Technology,

Thuwal 23955-6900, Saudi Arabia

${ }^{4}$ Department of Nanometrology, Wroclaw University of Science and Technology, Janiszewskiego 11/17, 50-372 Wrocław, Poland

${ }^{5}$ Institute of Experimental Physics, University of Wrocław, Maxa Borna 9, 50-204 Wrocław, Poland
\end{abstract}

\begin{abstract}
${ }^{a)}$ Author to whom correspondence should be addressed: ewelina.zdanowicz@pwr.edu.pl
ABSTRACT

Since the BGaN alloy is considered a promising material in the wide range of optoelectronic applications, a detailed study of its band structure and optical properties is highly demanded. Here, $\mathrm{B}_{x} \mathrm{Ga}_{1-x} \mathrm{~N}$ layers with $0.5 \%, 1.1 \%$, and $1.2 \%$ B were grown by metalorganic vapor-phase epitaxy on AlN/sapphire templates and investigated by structural and optical methods. The bandgaps of the investigated alloys were examined by contactless electroreflectance (CER) spectroscopy. Because no GaN layer is present in the investigated samples, the detected CER resonances do not overlap with the GaN-related signal, which is typical for BGaN layers grown on GaN templates. Thus, the energy of the bandgap-related transition in $\mathrm{BGaN}$ samples can be unambiguously determined from the resonances observed in the CER spectra. The boron-induced redshift of the bandgap was determined to be about $60 \mathrm{meV} / \%$ B for the studied samples. By means of photoluminescence measurements, the deteriorating optical quality of samples with increasing boron content is shown as the decreasing bandgapto defect-related emission intensity ratio. What is more, the defect-related emission is shifted from typical for GaN yellow range to the red and is located at $1.9 \mathrm{eV}$ for all $\mathrm{BGaN}$ samples.
\end{abstract}

Published under license by AIP Publishing. https://doi.org/10.1063/1.5140413

\section{INTRODUCTION}

Among the group of III-N ternary alloys, alloys of GaN and $\mathrm{BN}(\mathrm{BGaN})$ have become of interest due to their broad range of possible applications. So far, the beneficial influence of BGaN backbarrier on AlGaN/GaN HEMTs due to the enhancement of the two-dimensional electron gas confinement has been reported. ${ }^{1-4}$ Additionally, a decreased value of the refractive index for BGaN compared to $\mathrm{GaN}$ was reported, making the BGaN/GaN multilayer structures candidates for distributed Bragg reflectors. ${ }^{5,6} \mathrm{BGaN}$ alloys with specific B content are also thought to have a beneficial influence on GaN-based optoelectronic devices. In particular, a lattice match to the AlN and $\mathrm{SiC}$ substrates can be achieved for BGaN alloys containing $12 \%$ and $17 \%$ of boron, respectively. ${ }^{7}$
Furthermore, tuning the $\mathrm{B}$ content in the BGaN alloy up to $12 \%$ can lead to lattice matching to $\mathrm{AlGaN}$ in the entire aluminum range, ${ }^{8}$ therefore potentially enhancing the structural quality of the entire heterostructure. The quaternary $\mathrm{BInGaN}$ alloys are also predicted to maintain bandgaps similar to $\mathrm{InGaN}$ at a given indium concentration, meanwhile reducing the lattice mismatch to $\mathrm{GaN}^{9}$ This gives the opportunity to overcome the problem of efficiency drop at longer wavelengths in InGaN LEDs. $A b$ initio calculations predict zero heterointerface polarization for $\mathrm{BGaN} / \mathrm{BAlN}$ and BAlN/BGaN heterojunctions with a certain $\mathrm{B}$ content, ${ }^{10}$ which allows for the elimination of quantum-confined Stark effect present in GaN-based devices. Finally, the BGaN alloy is considered as a new material suitable for the application in UV-emitting devices. 
For zinc-blende $\mathrm{BGaN}$ and wurtzite $\mathrm{BGaN} / \mathrm{SiC}$ structures, the blueshift of the energy gap with increasing $\mathrm{B}$ content was predicted theoretically and observed experimentally. ${ }^{11-13}$ It was also shown that $\mathrm{BGaN}$ exhibits a similar optical gain to $\mathrm{GaN} .{ }^{14}$ However, for wurtzite phase $\mathrm{BGaN}$, the influence of boron on the bandgap of the alloy is not evident, ${ }^{15}$ since theoretical works predict blueshift of the bandgap, ${ }^{13}$ while experimental results show the opposite trend $^{8,16,17}$ for samples with boron content up to $5.5 \%$. This discrepancy may be explained by alloy fluctuations in experimentally examined samples and computational limitations concerning the simulation of low boron contents. ${ }^{18}$ Unfortunately, the GaN-related signal overlaps with the $\mathrm{BGaN}$-related signal for $\mathrm{BGaN}$ layers grown on $\mathrm{GaN}$ templates. Therefore, the proper determination of the bandgap energy in this kind of structure may be challenging.

In this work, BGaN samples with various B contents grown on sapphire substrates with AlN buffer by metalorganic vaporphase epitaxy (MOVPE) are studied. A detailed investigation of boron influence on the bandgap and photoluminescence (PL) in $\mathrm{GaN}$ is presented. The bandgap energy of $\mathrm{BGaN}$ alloys is studied by means of contactless electroreflectance (CER) spectroscopy, which has not been done for this alloy so far. This absorption-like technique is an appropriate tool for bandgap determination since it is not sensitive to localized states and reveals only transitions between delocalized states. ${ }^{19-21}$ It is revealed that the addition of boron slightly redshifts the energy gap of BGaN alloy compared to $\mathrm{GaN}$. Furthermore, the luminescent properties of BGaN alloys are studied showing that with increasing boron contents the bandgaprelated emission is quenched in favor of defect-related emission that is observed in the red and yellow spectral range.

\section{EXPERIMENTAL METHODS}

Examined samples consisted of approximately $350 \mathrm{~nm}$ thick $\mathrm{BGaN} / 390 \mathrm{~nm}$ thick AlN layers grown on a double-side polished c-plane sapphire by MOVPE in a 2-in. single-wafer horizontal reactor. ${ }^{22}$ The structures were grown at a low pressure of $10 \mathrm{kPa}$. Trimethylgallium, trimethylaluminum, tris(dimethylamino)borane, and $\mathrm{NH}_{3}$ were used as the sources of $\mathrm{Ga}, \mathrm{Al}, \mathrm{B}$, and $\mathrm{N}$, respectively. After thermal cleaning of the sapphire substrate under hydrogen ambient, the AlN layers were directly grown on sapphire substrates at $1080^{\circ} \mathrm{C}$, followed by $\mathrm{BGaN}$ growth at $900^{\circ} \mathrm{C}$. Hydrogen and nitrogen were used as carrier gases for $\mathrm{AlN}$ and $\mathrm{BGaN}$ growth, respectively.

X-ray rocking curves (RCs) and reciprocal lattice maps (RLMs) were obtained using the Panalytical Empyrean diffractometer equipped by the Pixcel3D detector and planar goniometer.

For CER measurements, samples were placed in a capacitor-like system between two electrodes, where the upper semi-transparent one is made of copper mesh and located about $0.5 \mathrm{~mm}$ above the sample surface. Electromodulation is achieved by applying an AC voltage to the electrodes. A single grating $0.75 \mathrm{~m}$ monochromator was used to disperse the light from a laser-driven xenon lamp. Light reflected from the sample was detected by a photomultiplier utilizing a lock-in technique. Temperature-dependent photoluminescence (PL) measurements were performed with samples mounted on a cold finger in a helium closed-cycle refrigerator coupled with a programmable temperature controller. A $325 \mathrm{~nm} \mathrm{He-Cd} \mathrm{laser} \mathrm{was}$ focused on the sample and used as the excitation source. The optical signal was detected by a Peltier-cooled CCD camera. Transmittance $(T)$ and reflectance $(R)$ measurements were conducted for the absorption edge examination. Light from a $150 \mathrm{~W}$ halogen lamp was dispersed by a single grating $0.3 \mathrm{~m}$ monochromator and transmitted through the sample. The output signal was detected by a GaP photodetector using a lock-in technique.

\section{RESULTS AND DISCUSSION}

For the XRD measurements, a $\mathrm{Cu}_{K \alpha 1}=1.540597 \AA$ radiation source was used. RC was obtained in a triple-axis diffractometer configuration via a $2 \theta / \omega$ scan. The curves were simulated to determine the composition of the layers. The lattice parameters $\mathrm{a}=2.5500(5) \AA$ and $\mathrm{c}=4.2154(10) \AA$ were used for the simulation based on PDF-4 Card No. 00-049-1327. The stiffness tensors' values for RC simulations were taken from Ref. 23. Based on RLM (01.5) [Fig. 1(a)], the relaxation of the BGaN layer was concluded. Taking into account the large lattice mismatch between $\mathrm{BGaN}$ and AlN and the significant thickness of the BGaN layer (exceeding the critical thickness), the relaxation degree close to $100 \%$ is deduced; however, the presence of small residual strain close to the BGaN/AlN interface cannot be excluded. Based on this assumption, the boron content was determined to be $0,0.5 \%, 1.1 \%$, and $1.2 \%$ for the four samples under study. Figure 1(b) depicts the (002) XRD diffraction pattern obtained from the $2 \theta / \omega$ scan for all investigated samples. The peak corresponding to the AlN template is visible together with the peak from the BGaN layer. Evidently, the $\mathrm{BGaN}$ peak position is shifted toward higher angles for samples with increasing boron content indicating the formation of a BGaN alloy. The basic indicator of the quality of the structure is the full (a)

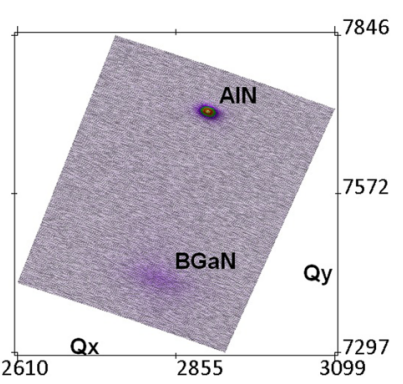

(c)

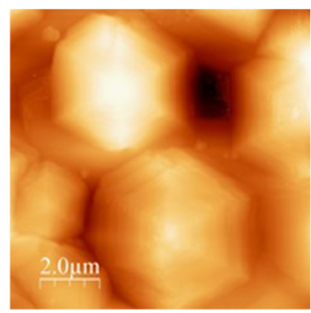

(b)

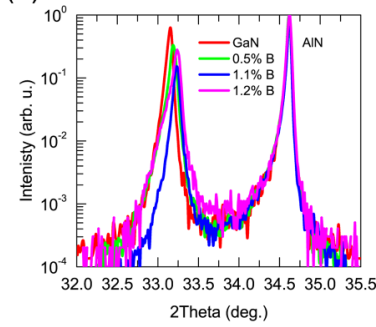

(d)

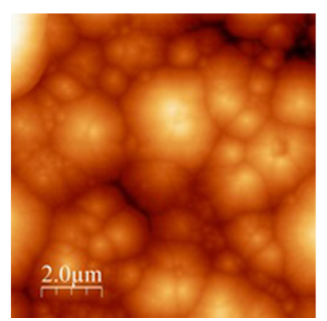

FIG. 1. (a) The (01.5) plane RLM of the structure containing 1.1\% B. (b) (002) XRD $2 \theta / \omega$ curves for all investigated samples. (c) and (d) AFM images of surface morphology for samples containing $0 \% \mathrm{~B}$ and $1.1 \% \mathrm{~B}$, respectively. 
width at half maximum (FWHM) of the measured reflection. Generally, the FWHM value consists of several factors, ${ }^{24-27}$ including instrumental broadening, broadening due to mosaicity (tilt and twist), and broadening due to lattice strain at dislocations. Here, the mosaicity factor is dominant and the other factors have negligible impact. Therefore, the FWHM values reported in Table I scale with the threading dislocation density in our samples. ${ }^{24-26}$ The value of the FWHM parameter increases with increasing boron content, indicating deteriorating structural quality. The highest value of the FWHM parameter was found for the sample containing $1.1 \%$ B indicating the lowest crystalline quality of this sample. The main contribution to broadening of the XRD peak originates from the lattice mismatch, but it also can be attributed to the alloying effect. ${ }^{24}$

Figures 1(c) and 1(d) show AFM images of surface morphology for reference $\mathrm{GaN}$ and sample containing $1.1 \% \mathrm{~B}$. The measured values of the root mean square (RMS) roughness parameter are $19,72,20$, and $122 \mathrm{~nm}$ for samples containing $0 \%, 0.5 \%, 1.1 \%$, and $1.2 \% \mathrm{~B}$, respectively. These high values of RMS originate from lattice mismatch present in our samples since $\mathrm{BGaN}$ layers were grown directly on the AlN template.

Figure 2 depicts CER and photoluminescence spectra together with the absorption edge measured at room temperature for samples containing $0.5 \%, 1.1 \%, 1.2 \% \mathrm{~B}$, and the reference GaN sample. For all samples, well-resolved CER resonances and PL peaks are visible. Lower quality of optical spectra obtained for $1.1 \%$ B sample results from its lower structural quality, which is consistent with the highest value of the FWHM parameter. The resonances visible in CER spectra redshift with increasing boron content. Since the CER method is sensitive only to optical transitions between delocalized states, ${ }^{21}$ this indicates the redshift of the bandgap with increasing $\mathrm{B}$ content. It is in accordance with previous reports for photoluminescence studies. ${ }^{8,16,17}$ For all samples, the photoluminescence signal associated with near band edge emission is present; however, it deteriorates with increasing $\mathrm{B}$ content. A similar trend was observed previously and was attributed to the increasing number of defects. ${ }^{8,16}$ Furthermore, the defect- to bandgap-related emission intensity ratio increases with increasing boron content as shown in Fig. 4(c). The PL peak energy is consistent with the bandgap energy established from CER within the experimental accuracy. The energies obtained from CER and PL are depicted in Fig. 3(b).

The values of the bandgap energies obtained from CER were extracted from fitting of the CER spectra with the well-known Aspnes formula, ${ }^{20}$

$$
\frac{\Delta R}{R}(E)=\operatorname{Re}\left[C e^{i \varphi}\left(E-E_{g}+i \Gamma\right)^{-m}\right]
$$

TABLE I. Full width at half maximum parameters of (002) reflection for all investigated samples.

\begin{tabular}{cc}
\hline \hline$\%$ B & FWHM $(\operatorname{arc~sec})$ \\
\hline 0.0 & 938 \\
0.5 & 1446 \\
1.1 & 1730 \\
1.2 & 1540 \\
\hline \hline
\end{tabular}

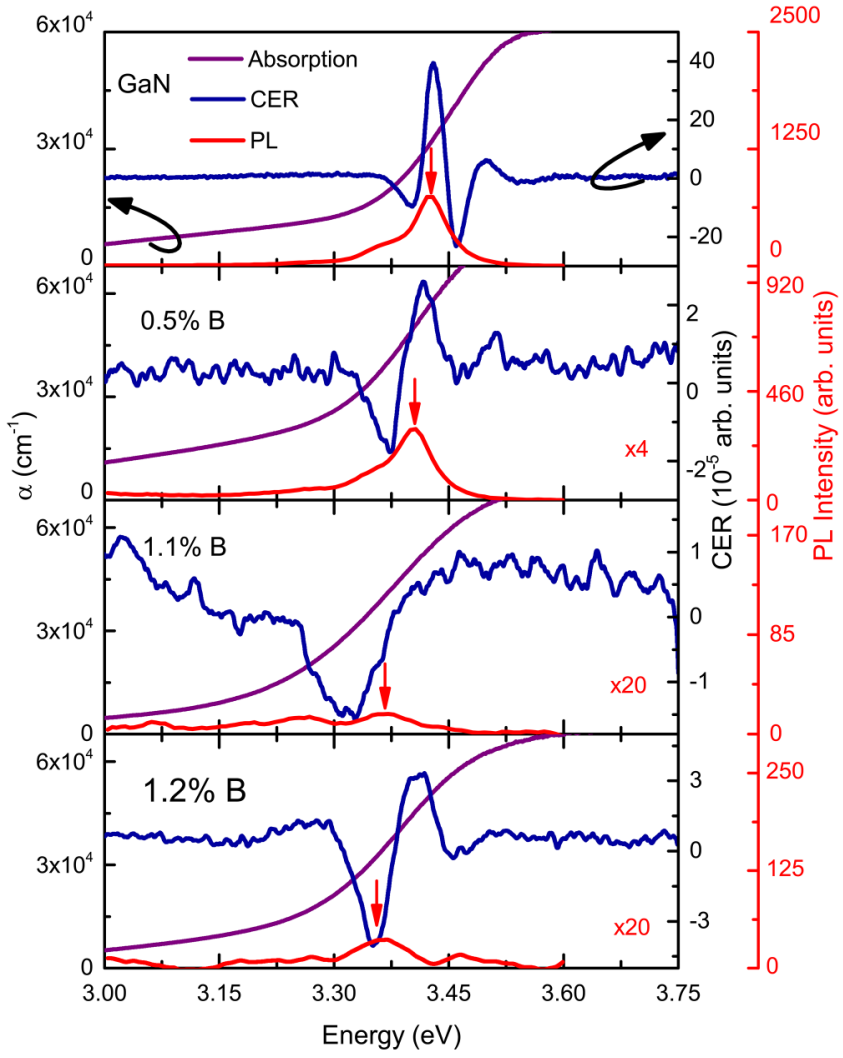

FIG. 2. Room temperature CER and PL spectra together with the absorption edge for samples with different $B$ contents.

where $C$ and $\varphi$ are the amplitude and phase of the signal and $E_{g}$ and $\Gamma$ are the energy gap and the broadening parameter of the transition, respectively. In this case, the value of 2.5 for $m$ parameter was adopted, which stands for a band-to-band transition. We note that no Fabry-Pérot oscillation interrupts the optical spectra since our BGaN layers of a significant thickness are grown on AlN and no GaN buffer layer is present underneath. This kind of sample architecture enables us to unambiguously assign the detected emission or resonance to optical transitions in the BGaN alloys without the possibility of confusion with spectrally close signal from GaN. On this basis, we conclude that the influence of boron on the energy gap in $\mathrm{GaN}$ is moderate because the energy gap of the alloy redshifts from the GaN energy gap by about $60 \mathrm{meV} / \% \mathrm{~B}$ in the investigated B range. This is in accordance with values reported previously ${ }^{17}$ for a similar $B$ content range. The observed tendency does not concur with the energy gap evolution predicted within the virtual crystal approximation considering the proper direct gap in wurtzite $\mathrm{BN}\left(13.2 \mathrm{eV}^{18}\right)$, thus indicating that BGaN is a highly mismatched alloy. ${ }^{21}$

Figure 2 illustrates the absorption spectra measured for all samples. They were obtained from transmission $(T)$ and reflectance 

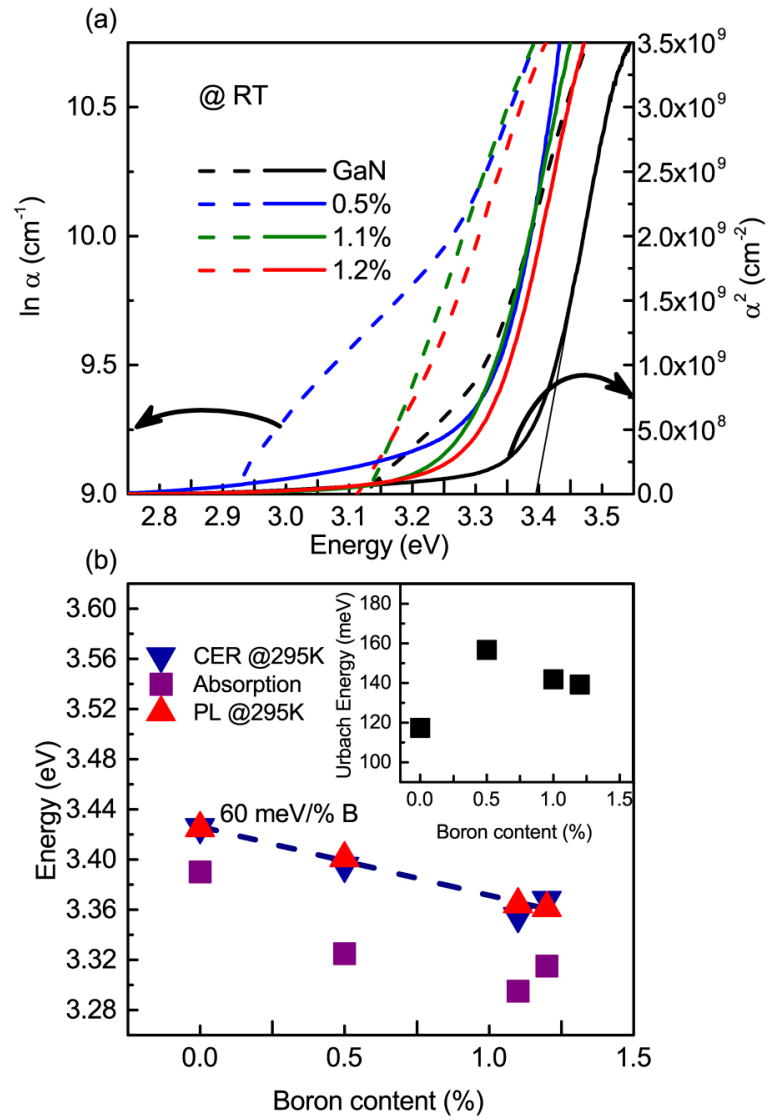

FIG. 3. (a) Optical absorption spectra for all investigated samples. (b) Bandgap energies obtained from PL, CER, and absorption measurements. The inset shows values of Urbach energies obtained from a linear fit of $\ln \alpha(E)$.

(R) spectra according to the standard formula, ${ }^{28}$

$$
\alpha=-\frac{1}{d} \ln \frac{T}{(1-R)^{2}},
$$

where $d$ is the BGaN layer thickness. The right-hand axis of Fig. 3(a) refers to the square of the absorption coefficient $\alpha^{2}(E)$. Since for the analyzed molar fractions of $\mathrm{B}, \mathrm{BGaN}$ is predicted to be a direct bandgap semiconductor, ${ }^{13,15,18}$ the linear extrapolation of $\alpha^{2}(E)$ determines the optical bandgap energy. Figure 3(b) shows that the bandgap energies obtained from absorption measurements are lower than those from CER and PL experiments; however, they reveal a similar shift with the $\mathrm{B}$ content. The left-hand axis in Fig. 3(a) refers to the natural logarithm of the absorption coefficient, $\ln \alpha$. The relationship between the $\ln \alpha$ and photon energy $E$ is given by Urbach's rule,

$$
\ln \alpha=\ln \alpha_{0}+\frac{E}{E_{U}}
$$

where $\alpha_{0}$ is a constant and $E_{U}$ is the Urbach energy. The linear dependence of $\ln \alpha$ vs photon energy $E$ indicates the absorption tail on the density of states inside the bandgap. The dependences plotted by dashed lines in Fig. 3(a) clearly follow this trend indicating absorption on states inside the bandgap rather than bandgap-related absorption. This fact can be attributed to defect states introduced in heteroepitaxial growth of our structures because of the significant lattice mismatch between the AlN buffer and the BGaN layer. In the CER experiment, the probed region is limited to the near-surface area. Conversely, light is transmitted through the entire structure in the absorption measurement, and, thus, the area with lower crystalline quality also contributes to the signal. For this reason, bandgap energies obtained from absorption measurements are undervalued and cannot be correctly specified. The values of $E_{U}$ obtained from linear fitting of $\ln \alpha$ are plotted in the inset of Fig. 3(b). As it can be seen, the values of the Urbach energy obtained for B-containing samples are higher than that for the GaN sample. This is a manifestation of the tail states formed as a consequence of alloying-induced lattice distortion.

The emission properties of analyzed BGaN samples have been investigated by PL measurements. Spectra obtained at low and room temperature are shown in the upper and lower panels in Fig. 4(a), respectively. The reference GaN spectrum measured at low (a)
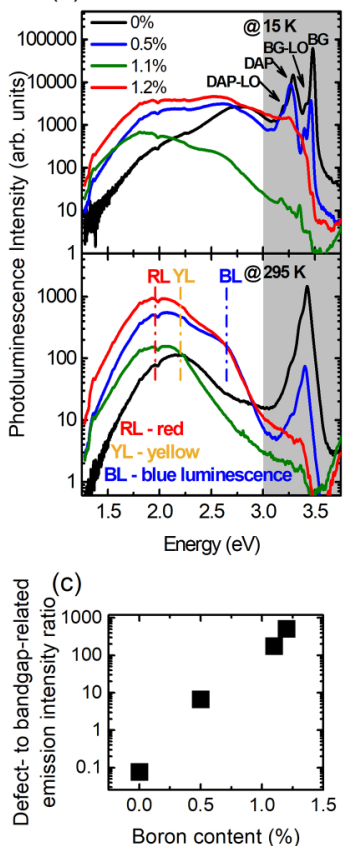

(b)

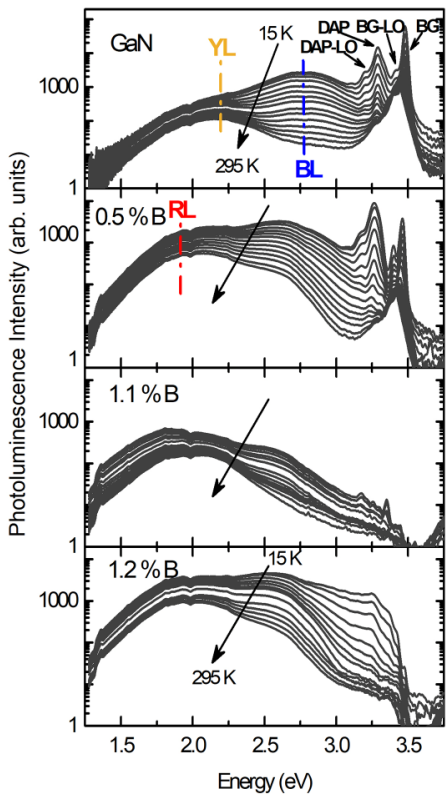

FIG. 4. Shortcuts $R L, Y L$, and $B L$ together with eye-guiding lines indicate the spectral positions of red, yellow, and blue luminescence lines, respectively. (a) Comparison of low and room temperature photoluminescence spectra for all investigated samples. (b) The evolution of the photoluminescence spectra from $15 \mathrm{~K}$ to $295 \mathrm{~K}$ for all investigated samples. (c) Red defect- to bandgap-related emission intensity ratio as a function of boron content at room temperature. 
temperature exhibits two distinct peaks above $3.0 \mathrm{eV}$. The highest energy peak at about $3.48 \mathrm{eV}$ is the bandgap-related emission followed by its phonon replica shifted by $97 \mathrm{meV}$. The peak located near $3.3 \mathrm{eV}$ associated with the donor-acceptor transition ${ }^{30-33}$ (DAP) is followed by its phonon replica (DAP-LO). For the sample with the lowest boron content $(0.5 \% \mathrm{~B})$, the same emission lines appear at spectral positions redshifted by about $20 \mathrm{meV}$. The DAP-LO peak is more redshifted (about $101 \mathrm{meV}$ ) from the DAP peak than in GaN sample indicating a larger LO phonon energy. This agrees with expectations because for the wurtzite phase $\mathrm{BN}$ LO phonon energy is about $159 \mathrm{meV}^{34}$ and the value of $92 \mathrm{meV}$ is reported for $\mathrm{GaN} .{ }^{35}$ For samples with $1.1 \%$ and $1.2 \% \mathrm{~B}$, a significant broadening of the defect-related bands prevents the proper detection of bandgap-related emissions. The lower panel of Fig. 4(a) shows the bandgap-related peaks for $\mathrm{GaN}$ and $\mathrm{B}_{0.005} \mathrm{GaN}_{0.995}$ samples at room temperature.

Figure 4(b) shows the evolution of the PL spectra as a function of temperature for all studied samples. Comparison of the first $(\mathrm{GaN})$ and the second $(0.5 \% \mathrm{~B})$ upper-most panels shows that the bandgap-related emission together with neighboring lower energy lines follows the same temperature dependence.

The defect-related bands for GaN are shown in the first upper panel in Fig. 4(b). At a low temperature, the most prominent defect band is a broad blue luminescence (BL) band. The intensity of this band continuously decreases with increasing temperature and the typical for GaN yellow luminescence (YL) dominates at room temperature. According to the literature, the $\mathrm{BL}$ band has been observed in MOVPE grown undoped GaN. It may arise from transitions from the shallow donors (in the case of low temperature) or from the conduction band (in the case of higher temperatures) to relatively deep acceptors. ${ }^{33}$ The YL band is one of the most studied defect bands in $\mathrm{GaN}$, but its origin still remains unclear. However, one plausible explanation is that $\mathrm{YL}$ is attributed to transitions to $\mathrm{V}_{\mathrm{Ga}}$-related defects bound to structural defects or complexes like $\mathrm{V}_{\mathrm{Ga}} \mathrm{O}_{\mathrm{N}}$ or $\mathrm{V}_{\mathrm{Ga}} \mathrm{C}_{\mathrm{N}}{ }^{33}$

Similar behavior is seen for B-containing samples; however, two different aspects appear. First is that for $0.5 \%$ and $1.2 \% \mathrm{~B}$ samples, the BL does not disappear completely at room temperature. Second is that the defect-related emission shifts from yellow to red for all B-containing samples [see lower panel in Fig. 4(a)]. After boron incorporation, a change in the concentration of native defects and a creation of new B-related defects, i.e., $B$ in the interstitial positions or $B_{N}$, are expected. These defects can be responsible for the red emission. What is more, increasing boron content leads to a further deterioration of the bandgaprelated emission in favor of defect-related emission, which is observed at low and room temperatures. This trend is highlighted in Fig. 4(c) as the increasing defect- to bandgap-related emission intensity ratio established as the ratio between the intensities of the red band and bandgap-related emissions at room temperature.

Considering the lattice mismatch, a lower optical quality of $\mathrm{BGaN}$ layers grown on $\mathrm{AlN}$ compared to $\mathrm{BGaN} / \mathrm{GaN}$ layers is expected. However, it was valuable to study BGaN/AlN structures since this kind of sample architecture allows to eliminate the spectrally close signal from GaN in CER and PL spectra.

\section{SUMMARY}

In conclusion, the influence of boron on the bandgap of BGaN was investigated by means of CER spectroscopy. The redshift of the energy gap for $\mathrm{BGaN}$ samples compared to $\mathrm{GaN}$ is about $60 \mathrm{meV} / \% \mathrm{~B}$ for samples with $\leq 1.2 \% \mathrm{~B}$. Temperature-dependent PL measurements show that the emission from the BGaN sample with the lowest $\mathrm{B}$ content follows a similar temperature dependence to $\mathrm{GaN}$; however, the bandgap-related emission is quenched in favor of defect-related emission for increasing boron content. In the boron-containing samples, the defect-related emission is dominated by the red luminescence band, while yellow luminescence is usually the most prominent defect band for GaN samples.

\section{ACKNOWLEDGMENTS}

This work was performed within the Grant No. TEAM TECH/2016-3/16 from the Foundation for Polish Science. At the Wroclaw University of Science and Technology, this work was supported by the SONATA grant from the National Science Centre (No. 2015/17/D/ST7/04081). This work was financially supported by the KAUST (No. BAS/1/1676-01-01).

\section{REFERENCES}

${ }^{1}$ M. Gassoumi, A. Helali, H. Maaref, and M. Gassoumi, Results Phys. 12, 302 (2019).

${ }^{2}$ L. Guenineche and A. Hamdoune, Mater. Res. Express 3, 055003 (2016).

${ }^{3}$ T. Han, H. Zhao, and X. Peng, Superlattices Microstruct. 126, 57 (2019).

${ }^{4}$ V. Ravindran, M. Boucherit, A. Soltani, S. Gautier, T. Moudakir, J. Dickerson, P. L. Voss, M.-A. di Forte-Poisson, J.-C. De Jaeger, and A. Ougazzaden, Appl. Phys. Lett. 100, 243503 (2012).

${ }^{5}$ S. Watanabe, T. Takano, K. Jinen, J. Yamamoto, and H. Kawanishi, Phys. Status Solidi C 0, 2691 (2003).

${ }^{6}$ M. Abid, T. Moudakir, Z. Djebbour, G. Orsal, S. Gautier, A. En Naciri, A. Migan-Dubois, and A. Ougazzaden, J. Cryst. Growth 315, 283 (2011).

${ }^{7}$ B. P. Gunning, M. W. Moseley, D. D. Koleske, A. A. Allerman, and S. R. Lee, J. Cryst. Growth 464, 190 (2017).

${ }^{8}$ J. Jurkevičius, J. Mickevičius, A. Kadys, M. Kolenda, and G. Tamulaitis, Physica B 492, 23 (2016).

${ }^{9}$ L. Williams and E. Kioupakis, Appl. Phys. Lett. 111, 211107 (2017).

${ }^{10}$ K. Liu, H. Sun, F. AlQatari, W. Guo, X. Liu, J. Li, C. G. Torres Castanedo, and X. Li, Appl. Phys. Lett. 111, 222106 (2017).

${ }^{11}$ T. Honda, M. Kurimoto, M. Shibata, and H. Kawanishi, J. Lumin. 87-89, 1274 (2000).

${ }^{12}$ L. Escalanti and G. L. W. Hart, Appl. Phys. Lett. 84, 705 (2004)

${ }^{13}$ A. Said, M. Debbichi, and M. Said, Optik 127, 9212 (2016).

${ }^{14}$ T. Honda, M. Shibata, M. Kurimoto, M. Tsubamoto, J. Yamamoto, and H. Kawanishi, Jpn. J. Appl. Phys. 39, 2389 (2000).

${ }^{15}$ T. Akiyama, K. Nakamura, and T. Ito, Appl. Phys. Express 11, 025501 (2018).

${ }^{16}$ A. Kadys, J. Mickevičius, T. Malinauskas, J. Jurkevičius, M. Kolenda, S. Stanionyte, D. Dobrovolskas, and G. Tamulaitis, J. Phys. D Appl. Phys. 48, 465307 (2015).

${ }^{17}$ A. Ougazzaden, S. Gautier, T. Moudakir, Z. Djebbour, Z. Lochner, S. Choi, H. J. Kim, J.-H. Ryou, R. D. Dupuis, and A. A. Sirenko, Appl. Phys. Lett. 93, 083118 (2008).

${ }^{18}$ M. E. Turiansky, J.-X. Shen, D. Wickramaratne, and C. G. Van de Walle, J. Appl. Phys. 126, 095706 (2019).

${ }^{19}$ Y. S. Huang and F. H. Pollak, Phys. Status Solidi A 202, 1193 (2005).

${ }^{20}$ R. Kudrawiec, Phys. Status Solidi B 247, 1616 (2010).

${ }^{21}$ R. Kudrawiec and W. Walukiewicz, J. Appl. Phys. 126, 141102 (2019). 
${ }^{22}$ K. Ohkawa, K. Nakamura, A. Hirako, and D. Iida, J. Cryst. Growth 516, 17 (2019).

${ }^{23}$ A. Bosak, J. Serrano, M. Krisch, K. Watanabe, T. Taniguchi, and H. Kanda, Phys. Rev. B 73, 041402 (2006).

${ }^{24}$ M. A. Moram and M. E. Vickers, Rep. Prog. Phys. 72, 036502 (2009).

${ }^{25}$ R. Wang, J. L. Zhang, R. X. Yang, and X. J. Zhang, Adv. Mat. Res. 433-440, 606 (2012).

${ }^{26}$ R. Chierchia, T. Bottcher, S. Figge, M. Diesselberg, H. Heinke, and D. Hommel, Phys. Status Solidi B 228, 403 (2001).

27. Serafinczuk, K. Moszak, L. Pawlaczyk, W. Olszewski, D. Pucicki, R. Kudrawiec, and D. Hommel, J. Alloys Compd. 825, 153838 (2020). .

${ }^{28}$ J. I. Pankove, Optical Processes in Semiconductors (Dover Publications, 1971).
${ }^{29}$ F. Urbach, Phys. Rev. 92, 1324 (1953).

${ }^{30}$ M. Leroux, N. Grandjean, B. Beaumont, G. Nataf, F. Semond, J. Massies, and P. Gibart, J. Appl. Phys. 86, 3721 (1999).

${ }^{31}$ S. C. Jain, M. Willander, J. Narayan, and R. V. Overstraeten, J. Appl. Phys. 87, 965 (2000).

${ }^{32}$ G. Li, S. J. Chua, S. J. Xu, W. Wang, P. Li, B. Beaumont, and P. Gibart, Appl. Phys. Lett. 74, 2821 (1999).

${ }^{33}$ M. A. Reshchikov and H. Morkoç, J. Appl. Phys. 97, 061301 (2005).

${ }^{34}$ N. Ohba, K. Miwa, N. Nagasako, and A. Fukumoto, Phys. Rev. B 63, 115207 (2001). .

${ }^{35}$ D. Kovalev, B. Averboukh, D. Volm, B. K. Meyer, H. Amano, and I. Akasaki, Phys. Rev. B 54, 2518 (1996). 